\title{
Pedacocías RuRALISTAS: CON LOS PIES EN URUGUAY Y LOS OJOS PUESTOS EN MÉXICO (1920-1960)
}

Rural Pedagogies: With the Feet in Uruguay and the Eyes on Mexico (1920-1960)

\section{Pamela Ruth Reisin}

Centro de Estudios Avanzados, Facultad de Ciencias Sociales,

Universidad Nacional de Córdoba. Argentina

pamela_reisin@yahoo.com.ar

\begin{abstract}
Resumen
Con el objetivo de reconstruir la configuración de la pedagogía ruralista en Uruguay entre 1930 y 1960, aquí se centra la mirada en el documento periodístico Síntesis del proceso educacional mexicano (1938), elaborado por el maestro y periodista uruguayo Julio Castro, contextualizándolo en la vida y obra de su autor. El texto hace referencia a las reformas de la educación rural en el México posrevolucionario, pero, lejos de ser un frío informe estadístico, expresa aspiraciones, comparaciones y reflexiones del autor. Para Castro mirar a México era una forma de repensar su país y la educación en éste. La metodología de las "historias conectadas" nos permitirá percibir cómo las conexiones entre México y Uruguay estaban entramadas en la configuración de la pedagogía ruralista uruguaya.
\end{abstract}

Palabras clave: pedagogía ruralista, Uruguay, México, Julio Castro.

\section{Abstract}

With the aim of reconstructing the configuration of the rural pedagogy in Uruguay from 1930 to 1960, this paper analyzes the journalistic document Síntesis del proceso educacional mexicano (1938), wrote by Uruguayan teacher and journalist Julio Castro. The article contextualizes the document within the author's life and work. The Síntesis refers to the reforms on rural education of post-revolutionary Mexico; however, far from being a cold statistical report, the text expresses the author's aspirations, comparisons and reflections. For Castro, looking at Mexico was a way to reflect upon his country and its education system. The use of the "entangled history" methodology will allow us to show how the connections between Mexico and Uruguay were interwoven in the formation of the Uruguayan rural pedagogy. 
Keywords: rurali pedagogy, Uruguay, México, Julio Castro.

\section{Introducción}

Las metodologías de investigación de historias conectadas o cruzadas son un ámbito en exploración en la historia de la educación; si bien existen investigaciones de historia regional y comparada, el estudio de las conexiones entre distintos proyectos y experiencias educativas son incipientes. En los últimos 15 años investigadores, principalmente de Brasil, México y Argentina (Civera y Rico, 2018; Civera, Alfonseca y Escalante, 2011; Civera y Lionetti, 2010; Werle, 2007) coordinaron publicaciones de artículos sobre historia de la educación de los campos, con la intención de avanzar en análisis comparativos que den cuenta de las singularidades y de los aspectos comunes entre diversos procesos de escolarización en el ámbito rural en América Latina.

Identificar proyectos educativos comunes permite cuestionar la noción de procesos aislados y autogenerados exclusivamente por lo local o lo nacional y da cuenta de los diversos medios de circulación de ideas y de experiencias pedagógicas entre distintos territorios. No existe la copia directa ni la imposición total, siempre hay intersticios para que los actores implicados realicen adaptaciones, recontextualizaciones, resistencias e invenciones. En este sentido, Civera y Rico (2018) sostienen que las interconexiones en la educación de los campos fueron multidireccionales, "no solo de lo urbano hacia lo rural y de Europa hacia otras regiones", e invitan a investigarlas señalando ejemplos de interrelaciones entre proyectos educativos e ideas pedagógicas desarrolladas en distintos lugares del mundo a lo largo del siglo XX.

Asumiendo este desafío investigativo consideramos que la propuesta metodológica de historias conectadas puede ser una herramienta potente para el análisis del proceso de configuración de la pedagogía ruralista uruguaya (1930-1960) ya que, sostenemos, a modo de hipótesis, que diversas conexiones que trazaron distintos maestros uruguayos con experiencias y proyectos educativos mexicanos generaron aportes significativos en la configuración de una pedagogía ruralista en Uruguay. Resulta necesario mencionar que, hasta el momento, la historiografía de la educación uruguaya no ha investigado específicamente el vínculo con México. Consideramos que este es un eje potente de indagación, teniendo en cuenta las

\footnotetext{
1 "Instituciones no necesariamente escolares, como las misiones pedagógicas y educativas de España, Chile, México, Uruguay y Brasil tuvieron un auge especial en las zonas rurales y a partir de su éxito se trasladaron y adecuaron a las áreas urbanas. Las interconexiones entre distintas ideas y experiencias pedagógicas y sus influencias recíprocas son un campo aún por explorar en la Historia de la Educación. La literatura existente sugiere que las influencias tuvieron múltiples direcciones, y no solo de lo urbano hacia lo rural y de Europa hacia otras regiones" (Civera y Rico, 2018:23).
} 
distintas referencias (explícita e implícita) que hay de la educación rural mexicana en los escritos, experiencias y programas pedagógicos elaborados por maestros uruguayos entre 1930 y 1960. Estas conexiones, lejos de ser un caso aislado, se inscriben dentro de un entramado histórico que comenzó a gestarse a comienzos del siglo xx; ya para las décadas de 1920 y 1930, la idea de América Latina como entidad trasnacional circulaba por los ámbitos intelectuales de una generación preocupada por el devenir de sus países y de la región; y, sin duda, un acontecimiento que generó una gran conmoción en la región fue la Revolución mexicana y el proceso posterior de reorganización social y política en dicho país. El interés de los maestros uruguayos por la educación mexicana no fue casual, siendo a su vez la política exterior impulsada por la mayoría de los gobiernos mexicanos proclives a estos intercambios y a la recepción de intelectuales exiliados de distintos países y así como también a la difusión de sus proyectos políticos y culturales en el escenario internacional (Moraga, 2018 y Pita, 2014). Puntualmente, las conexiones entre México y Uruguay en materia educativa han sido diversas entre 1930 y 1960, pero para fundamentar esta hipótesis señalaremos en este artículo los intercambios centrales y sus aportes en la configuración de la pedagogía ruralista en Uruguay.

Con este recorrido se pretende describir las conexiones que trazó el Movimiento en Favor de la Educación Rural (Soler, 1987) uruguayo con experiencias y concepciones pedagógicas de la educación rural mexicana, y situar en este proceso el accionar de uno de sus principales exponentes, el maestro y periodista uruguayo Julio Castro (1908-1977), sobre quien se centrará particularmente en este artículo. Se analizará su trayectoria poniendo el énfasis de su intervención en la circulación de ideas y experiencias pedagógicas entre Uruguay y México. Para ello, se profundizará en el análisis de un texto de su autoría, Síntesis del proceso educacional mexicano, el cual es uno los primeros documentos donde comparó a México con Uruguay en materia educativa y se preguntó por las posibilidades de construir experiencias similares en su país. Por último, se retomará lo planteado sobre configuración de la pedagogía ruralista de Uruguay y se reconstruirá la trayectoria de Castro y su vínculo con México en las décadas de los cuarenta, cincuenta y sesenta del siglo pasado, procurando identificar los aportes y las huellas de estas conexiones en la configuración de la pedagogía ruralista uruguaya.

\section{Las escuelas de los campos y los proyectos pedagógicos ruralistas}

Con el desarrollo de los sistemas educativos a fines del siglo XIX y principios del siglo XX se crearon escuelas públicas primarias en zonas urbanas como en áreas rurales. La cantidad, sentido y distribución de las escuelas en los campos varió en cada país en función del 
proyecto político y económico de las élites gobernantes, como también de la visión que éstas tenían sobre los habitantes de los territorios no urbanizados.

La composición de la población de los campos y la distribución de la propiedad de la tierra durante las primeras décadas del siglo xx eran heterogéneas en América Latina, y eso complejiza plantear una definición de una única ruralidad. Lo rural, como construcción cultural e histórica se configuró a partir del desarrollo de su opuesto: lo urbano. Esta distinción ha servido para clasificar a la población que se le asocia, asignándole distintos lugares en la estratificación social y en la administración pública. Para esta última, "lo rural suele ser definido por los censos en términos de densidad poblacional y su asociación a la ocupación en actividades del sector primario, lo cual tiene implicaciones en la asignación de recursos, establecimiento de programas de desarrollo, y muchos otros aspectos" (Civera y Costa Rico, 2018: 11).

La educación pública en el medio rural, haya tenido o no un programa diferenciado de las escuelas urbanas, siempre mantuvo como característica preponderante la inestabilidad debido a los conflictos políticos, las crisis económicas y las migraciones, es decir que "la ruralidad se hace presente en la escuela y transforma y crea proyectos pedagógicos" (Civera y Rico, 2018: 20-21). El concepto de "pedagogías ruralistas" sirve para englobar prácticas, concepciones, programas y políticas educativas que sostenían que la educación en el medio rural debía adquirir una orientación específica, diferenciada de la educación de las grandes urbes. Las mismas tuvieron su mayor desarrollo durante la primera mitad del siglo Xx; fueron propuestas educativas para los habitantes del campo, constituyeron una apuesta a la vida en ese contexto y por lo tanto buscaron brindar conocimientos que respondan a las necesidades de quienes allí viven y trabajan; sus actividades educativas excedieron los muros del local escolar e incluyeron tanto a niños y niñas como a jóvenes y a adultos. Vale aclarar que esta es una categoría teórica a posteriori y no una denominación histórica creada por los sujetos involucrados.

Las pedagogías ruralistas, si bien buscaron responder a las necesidades que quienes las llevaban a la práctica consideraban que tenían los habitantes de los campos, terminaron privilegiando la formación manual y práctica para el medio rural, quedando así la formación intelectual exclusivamente para las clases medias y altas de las ciudades. Esta diferenciación es uno de los cuestionamientos principales a estas pedagogías (Civera, 2011). La opción de una educación práctica estaba sustentada en las teorías pedagógicas en boga en el periodo entre guerras, principalmente el Movimiento Internacional de Escuela Nueva, la cual criticaba a la educación autoritaria, libresca y magistrocéntrica. Otra de las inspiraciones para las pedagogías ruralistas de América Latina fue la rusa, primero con Tosltoi (Moraga, 2016) y después con la Unión Soviética y la educación politécnica, que concebía al trabajo como un eje central para formar un "Hombre Nuevo" y una nueva sociedad (Civera y Rico: 2018).

En el caso uruguayo el surgimiento de las pedagogías ruralistas se generó, en sus inicios, como una estrategia para frenar el desplazamiento de población rural a los centros urbanos 
e incentivar la producción agrícola. Allí, la pregunta por la formación del "campesino" fue planteada cuando este modo de vida entró en crisis, es decir, el "campesino como sujeto de la educación comienza a tener visibilidad en el momento que aparece un problema social: la emigración del campo a la ciudad y el consiguiente despoblamiento del campo" (Romano, 2006). La categoría de campesino en Uruguay no implicaba una diferencia étnica, aunque sí cultural y social, ya que los modos de vida de los habitantes del campo eran distintos de los urbanos. En cambio, en el caso mexicano, no sólo la mayoría de la población vivía en el campo, sino que una gran proporción era indígena, lo cual agregaba no sólo diversidad cultural y étnica sino también lingüística (Calderón, 2018); no hablar castellano también implicaba una dificultad adicional en el proceso de alfabetización.

Durante los primeros años de la década de 1930, con la crisis del capitalismo a nivel mundial y del sistema agroexportador, se intensificó el proceso de migración interna en distintos países de la región. Los avances tecnológicos, científicos y económicos se concentraron principalmente en las grandes ciudades, marcando más aún las desigualdades entre los espacios rurales y urbanos. En Uruguay (Santos, 2018) y Brasil (Werle y Carvalho, 2009), durante las décadas de 1930 y 1940, se organizaron congresos y conferencias en las cuales los educadores debatieron sobre cómo evitar la despoblación de los campos y qué rol podía jugar la escuela en ese proceso; esto también se replicaba a nivel internacional, en la Conferencia de Educación convocada por el Bureau International d’Educatión realizada en Génova 1938 se analizó el fenómeno migratorio a nivel mundial y se defendió la ruralización de la educación en los campos para una estrategia para enfrentarlo.

Esta preocupación trasnacional da cuenta de que las pedagogías ruralistas no fueron un experimento educativo aislado, sino un proyecto de modernización en el cual se comenzaron a plantear relaciones entre la educación en el medio rural y el desarrollo económico local, ${ }^{2}$ ampliando así los sentidos y prácticas de la educación escolar y del trabajo docente. ${ }^{3}$

2 "Los proyectos ruralistas desplegados en América Latina, junto con otros similares armados en varios países como China y Turquía [...] constituyeron la base de una serie de políticas que tuvieron el deseo de que la escuela [rural] dejara de ser la «cenicienta» tradicional, para ser en cambio una preciosa mediación al servicio del desarrollo local. Hacia ahí se encaminaron los primeros proyectos piloto de la UNESCO desde su nacimiento y su formalización en la "Educación fundamental" (Civera y Costa Rico, 2018:18).

${ }^{3}$ En Uruguay "[...] el 'movimiento a favor de la educación rural' [...] resignificó aquel papel de la escuela rural en la sociedad desde su vertiente social, denunciando la situación de expandida pobreza que iba sembrando a su paso la modernización excluyente del agro. Pese a los intentos de una modernización incluyente, durante el período de industrialización por sustitución de importaciones, donde el paradigma de la 'agriculturización' procuraba por su lado abastecer a la industria, otorgándole a la agricultura familiar un papel estratégico en el autoabastecimiento del mercado interno. La experiencia de las 'escuelas granjas' fue un emergente de la tensión entre los dos modelos de desarrollo rural, formando al ciudadano y al agricultor, explorando alternativas a las establecidas por el modelo agroexportador basado en el latifundismo del hambre" (Díaz y Díaz, 2019: 114) 


\section{El Movimiento a favor de la Educación Rural uruguayo y la configuración de una nueva pedagogía}

En América Latina, México fue uno de sus principales promotores de la pedagogía ruralista, que constituyó en ese país una política de Estado implementada por los gobiernos federales posrevolucionarios (1920-1940) como una estrategia para ampliar su poder territorial en todo el país y fomentar el desarrollo de las áreas rurales, mediante políticas sociales y económicas, como lo fue la reforma agraria (Rockwell, 2005).

Las investigaciones historiográficas sobre educación rural en el México posrevolucionario (1920-1940) son numerosas y cada una hace un aporte desde una perspectiva particular (cultural, política, local, regional, nacional). Como trabajos ya clásicos es necesario mencionar a Loyo (1999), Civera (2008), Vaughan (2000), Rockwell (2007), Palacios (1999); mientras que en los últimos años destacan las investigaciones de Moraga (2018) y Calderón (2018) sobre este periodo, el primero hace referencia sobre todo a la figura de Vasconcelos y sus sucesores y el segundo problematiza el pasaje de la "educación indígena" a la "rural".

En Uruguay la investigación académica sobre este proceso está en una etapa más incipiente en comparación con la desarrollada por la academia mexicana. Los primeros trabajos fueron Angione et al. (1987) y Demarchi y Richero (1999); y más recientemente han publicado sobre el tema Romano (2006), Santos (2012 y 2018), Alonso y Scagliola (2012). Lo relevante aquí es que se cuenta con distintas fuentes primarias (textos escritos por los maestros que protagonizaron este proceso, siendo los más prolíficos Julio Castro y Miguel Soler Roca, además de que existe registro de experiencias, propuestas pedagógicas, actas de congresos de maestros y de inspectores, programas escolares, etc.) que permitirían avanzar en la reconstrucción historiográfica de este periodo y de esta pedagogía.

Al igual que el resto de los países de la región, a principios del siglo xx Uruguay tenía su economía basada en el modelo agroexportador sostenido por la explotación de grandes extensiones de tierra y el empleo de un número reducido de jornaleros. La producción extensiva de la ganadería hizo que muchos campesinos se quedaran sin tierra, y formaran precarias viviendas en los bordes de las haciendas, conformando "rancheríos"; otros migraron a las ciudades y quienes conservaron sus tierras acabaron viviendo en pequeños poblados, aislados unos de otros. Allí, el desarrollo de la pedagogía ruralista fue impulsada por maestros que vivían y trabajaban en esos territorios. Ellos conocían y padecían las malas condiciones de vida al igual que los demás habitantes, y presenciaban la despoblación del campo. Debido a estas urgencias algunos maestros ejercieron presión política, primero de forma individual y después colectiva, para que se habilitara el desarrollo de diferentes experiencias en el ámbito de la educación pública tendentes a transformar esta situación, buscando poner la escuela primaria al servicio del "desarrollo rural", concebido desde una 
perspectiva integral y no sólo económica. ${ }^{4}$ Estos maestros conformaron una organización social en la cual también participaron estudiantes de magisterio e inspectores escolares, denominada posteriormente por uno de sus protagonistas como Movimiento a favor de una nueva escuela rural (Soler, 1987). ${ }^{5}$ Dicha formación social no fue hegemonizada por ningún partido político, su composición ideológica era diversa, siendo algunos maestros cercanos a líneas progresistas dentro del Partido Nacional Independiente y del Partido Colorado, mientras que otros estuvieron vinculados con el Partido Comunista. Su relación con el Estado fue variando en función de quienes estuvieron en el poder, pasando de estar en la resistencia, principalmente en las décadas de 1930 y 1960, a ocupar cargos en el Consejo de Educación y motorizar y acordar de conjunto con el gobierno políticas educativas durante parte de las décadas de 1940 y 1950. Al respecto, Soler Roca señala que, el "progreso" en la "pedagogía nacional" que se logró "durante los años cuarenta y cincuenta" se debió principalmente a "la llegada a cargos directivos de educadores de talla; en otros momentos, la influencia y visión de quienes ocupaban los puestos superiores del cuerpo nacional de inspectores" y "la reflexión de pedagogos capitalinos y, como una fuerza sostenida y creciente, el empuje de un par de generaciones de educadores de base que supieron pasar de la denuncia a la propuesta, de la propuesta a la acción [...] a aquel proceso de dos décadas lo he denominado movimiento en favor de la educación rural" (Soler 1996: 394).

Este movimiento se generó en la década de 1930 a partir de cuestionamientos a las formas instituidas sobre lo escolar en el ámbito rural que realizaron estos maestros e inspectores, y desde ese cuestionamiento gestó propuestas pedagógicas que albergaban una nueva forma de concebir la escuela y su relación con el contexto y con los sujetos que en él habitan, así como también transformó las prácticas docentes, ya que maestros rurales, motorizados por un gran compromiso social y político, generaron nuevas prácticas de enseñanza, teniendo como fin "esencial", según el Programa de Escuelas Rurales, "crear las posibilidades que permitan el desarrollo integral del educando. En los distintos medios debe tender a compensar los déficit que nieguen posibilidades a ese desarrollo". ${ }^{6}$ Esta acción "compensadora" iba desde buscar generar soluciones mediante la organización colectiva con los habitantes de esa "comunidad" a la "desnutrición", a generar actividades lúdicas para que

${ }^{4}$ Soler Roca definía el "desarrollo rural" como "un proceso de comunicación humana de objetivos transformadores. Las acciones micro y aquellas desprovistas de fundamentos necesariamente políticos no tienen ninguna perspectiva de éxito" y consideraba que "No hay desarrollo rural sin organizaciones de base, cuyos miembros sientan la necesidad de una visión en cierto grado compartida de sus problemas, recursos y proyectos de producción y de vida" (Demarchi y Richero, 2014: 177 y 178).

${ }^{5}$ La noción de "movimiento" ha sido adoptada por Santos (2012) y Martinis y Redondo (2006) refiriéndose todas al mismo proceso histórico que implicó la generación y desarrollo de la pedagogía ruralista en Uruguay entre 1930 y 1960.

${ }^{6}$ Consejo Nacional de Enseñanza Primaria y Normal, 1950. 
niños trabajadores pudieran tener también tiempo para el juego. El auge de este movimiento comenzó a decaer cuando a comienzos de 1960 las "autoridades nacionales y educacionales adoptaron políticas tendientes al desmantelamiento de todo lo construido" (Soler, 1996: 394). Las elecciones en 1958 dieron por ganador al Partido Nacional, de tendencia conservadora, que en pocos años desarticuló todas las instituciones educativas destinadas a la educación rural creadas durante los gobiernos anteriores. Sin embargo, los alcances de las concepciones pedagógicas de este movimiento trascendieron el ámbito de la educación rural, "El sistema educativo en su conjunto no permaneció ajeno a este movimiento pedagógico gestado por los propios maestros y, en gran parte, emana de él el prestigio que caracterizó por décadas a la escuela pública uruguaya" (Demarchi y Richero, 1999: 10).

La preocupación por la educación en el campo era un tema común en el contexto latinoamericano en la primera mitad del siglo xx. Cuando en Uruguay los maestros comenzaron a debatir sobre la "ruralización de la educación", a mediados de la década de 1930, en México la educación rural ya tenía más de diez años de desarrollo, y bajo el gobierno de Cárdenas y con la expansión de la reforma agraria había tomado un nuevo impulso. Por ello, para quienes apostaron al desarrollo de esta pedagogía en Uruguay cobró importancia el ejemplo mexicano con las Misiones Culturales, las Normales Rurales y las escuelas Casa del Pueblo. En este sentido, es posible postular que la pedagogía ruralista la construyeron con los pies en Uruguay y los ojos en México posrevolucionario mediante la recontextualización y recreación que hicieron de estas experiencias educativas, en función tanto de sus propios intereses como de las posibilidades de acción que tenían en un país sustancialmente diferente del mexicano. Como hipótesis planteo que la configuración de la pedagogía ruralista uruguaya, y sus conexiones con la educación rural mexicana, puede ser analizada en tres etapas: fundacional (1933-1944), experimental (1945-1955) y de institucionalización (1956-1961) cuya descripción se abordará en un próximo artículo.

\section{Julio Castro, maestro y periodista latinoamericanista}

Uno de los maestros que ofició de nexo entre ambos países fue Julio Castro quien tendió puentes para que otros educadores uruguayos pudieran conocer los proyectos educativos mexicanos, sea viajando a dicho país o mediante la difusión de experiencias educativas mexicanas en escritos pedagógicos y notas periodísticas y en intervenciones públicas (congresos, conferencias, clases, etc.). Desde sus comienzos Castro combinó el periodismo con su compromiso político como educador. Entre 1934 y 1943 fue director de Escuelas 
Comunes y de Práctica en Montevideo y profesor de metodología en los Institutos Normales, y entre 1936 y 1939 (Soler, 2007). Como periodista y docente escribió sobre las problemáticas sociales que le que preocupaban e interesaban, y, las novedosas experiencias en educación rural que se desarrollaron en el México posrevolucionario; quizás por haber nacido en el campo y haber sido alumno de una escuela rural en el Departamento de Florida (Uruguay) se constituyó en un tema de su interés. El texto que aquí se analizará es la Síntesis del proceso educacional mexicano (Castro, 1938), uno de los cinco "Folletos Publicados por la Oficina de Prensa de Periodistas Libres", impreso en los Talleres Gráficos Sur de Montevideo.

En la década de 1930 la prensa estaba mayormente dominada por el gobierno autoritario de Terra y había pocos periodistas de oposición o independientes (Betemps, 2003). Gabriel Terra, político miembro del Partido Colorado y originariamente Batllista, quien había sido electo presidente en 1931, dos años después de su asunción, realizó un autogolpe de Estado aprovechando la crisis económica y la distracción generada por la disputa al interior de los partidos políticos. Mediante este golpe disolvió el Parlamento y el Consejo Nacional de Administración e implantó una dictadura, totalmente civil, que no por ello se abstuvo de encarcelar, deportar, incluso torturar a los opositores (Ansaldi y Giordano, 2012). En 1934 reformó la Constitución a su favor y logró ser elegido nuevamente, mandato que ejerció hasta junio de 1938. Lo sucedió Alfredo Baldomir Ferrari hasta 1942, periodo conocido como el "Golpe Bueno", ya que se volvió a reformar la Constitución, lo cual permitió que hubiera elecciones libres y democráticas, resultando ganadora una fórmula batllista del Partido Colorado.

Es posible que Castro, en 1938, con la publicación de la Síntesis, documento periodístico no circunscrito al ámbito académico sobre la educación en México, haya procurado instalar en la opinión pública la posibilidad de generar transformaciones sociales, políticas y económicas en un país que, anteriormente, en los gobiernos del llamado Impulso Batllista (19031916) había adoptado políticas progresistas en términos sociales y educativos, pero en ese entonces estaba sumergido en un clima político conservador. A su vez, dar a conocer en Uruguay qué pasaba en México en torno a la educación en el medio rural, tenía una motivación personal para él, un anhelo por comprender por qué en México hubo un desarrollo tan grande en este ámbito y la pregunta sobre qué podía aportar dicha experiencia en su país. Esta motivación se puede entrever en las comparaciones y reflexiones que hacía al respecto. Tres fueron sus preocupaciones centrales: la relación entre las estructuras sociales de las poblaciones rurales y las propuestas educativas, los proyectos para la "ruralización" de la educación; y la participación popular en el sostenimiento y desarrollo de procesos educativos. Estas temáticas se convirtieron en una constante inquietud para Julio Castro a lo largo de su vida. 
La Síntesis del proceso educacional mexicano, por la información que brinda en sus 29 páginas, da cuenta de una búsqueda bibliográfica extensa por parte de su autor, basada sobre todo en publicaciones de la Secretaría de Educación Pública (SEP), ${ }^{7}$ lo cual hace preguntarnos de qué modo tuvo acceso a esa documentación y cuáles eran las vías de comunicación e intercambio de información de esta índole en esos años. Una respuesta posible a este interrogante es que haya obtenido esa información a través del temprano vínculo que su colega Carlos Quijano mantuvo con funcionarios e intelectuales de dicho país.

Quijano, abogado, periodista y profesor universitario, tuvo una gran trascendencia en la formación del pensamiento latinoamericanista en el Uruguay (Espeche, 2016). Fue presidente del Centro Ariel siendo aún estudiante universitario; una vez recibido de abogado viajó en 1923 a París para formarse en economía y ciencias políticas. Ahí tuvo contacto con el argentino José Ingenieros y junto con otros estudiantes conformaron la Asociación General de Estudiantes Latinoamericanos (ACELA). En una de las actividades organizadas por la Asociación conoció al, por entonces, exsecretario de Educación Pública, José Vasconcelos quien estaba en Europa tras haber dejado su cargo por su enfrentamiento con el presidente Calles.

Con AGELA, además, realizó en París manifestaciones públicas de apoyo al gobierno de México, país que atravesaba un proceso de reorganización nacional después de 10 años de guerra civil y mantenía una relación conflictiva con los Estados Unidos. El gobierno mexicano se enteró de estas manifestaciones e invitó a Quijano e Ingenieros a visitar México, país por el cual estuvieron viajando durante tres meses en 1925. Cincuenta años después, sus relaciones con la intelectualidad mexicana fueron claves para que Quijano, ya anciano, pudiera exiliarse en este país cuando la dictadura militar azotaba Uruguay. Es probable que este inicial vínculo de Quijano con distintos políticos e intelectuales mexicanos haya servido para que los documentos de la SEP Ilegaran a Castro. Desde la década de 1930 Castro y Quijano fueron entrañables compañeros en la actividad periodística y en la militancia política, ambos allegados al Partido Nacional Independiente y fundadores, en 1939, del reconocido Semanario Marcha.

\section{La escuela rural debía ruralizarse}

El contenido de la Síntesis del proceso educacional mexicano está organizado en 15 apartados: (mayúsculas del original) "Las razas", "Estado social y cultural", "El problema del

\footnotetext{
7 Para realizar esta Síntesis, Castro se valió de publicaciones oficiales del gobierno mexicano, que consignó al pie de cada página de la siguiente manera: "Puig Casauranc. — La Instruc. Pública en Méjico a través de los Mensajes Presidenciales; El Maestro Rural” (revista) 15 de noviembre de 1934 (México); Emilio Portes Gil. "La Escuela y el
} 
indio", "La Revolución", "La instrucción pública bajo Porfirio Díaz", "El esfuerzo económico", "La Reforma Constitucional", "La Escuela Rural", "Actividades", "Los maestros", "Las Escuelas Normales Rurales Regionales", "Hacia la ruralización", "Las Misiones Culturales" y "Otras actividades educacionales". A continuación, se analizarán principalmente aquellos apartados en que Julio Castro utilizó el estudio del caso mexicano para pensar proyectos educativos en su país.

El documento comienza con una definición sobre la "actual escuela mexicana"; en ella el autor aclaraba que su carácter de "socialista" no era en el sentido "filosófico o político" sino en cuanto a la finalidad social de la escuela: "Es socialista en cuanto es una escuela para la sociedad, estructurada según las necesidades y los fines que de ella espera el movimiento social mexicano; ha sido realizada a la medida de las adaptaciones que tuvo que sufrir y de los obstáculos que tuvo que vencer" (Castro, 1938: 4); no obedeció a ningún plan o doctrina, sino que se fue haciendo a sí misma a la par que se iba construyendo el Estado posrevolucionario. Para Castro el movimiento ligado a la educación rural en México era el más "interesante" de su época y, según el autor, el estudio de estas realizaciones educacionales no podía hacerse de forma escindida de un análisis de la "realidad social, étnica, económica e histórica del país", tarea a la cual dedicó en este documento, en el que fue introduciendo comparaciones, reflexiones, críticas y propuestas para la educación rural en su país.

Una de las principales diferencias que Castro encontró entre el campo uruguayo y el mexicano tiene que ver con la forma de la distribución de la población rural en ambos países. " "Esta distribución de la población es muy importante para la solución de los problemas educacionales. Mientras en la hacienda o el ejido la escuela puede ser el centro social del poblado, en los lugares donde la población está dispersa, el problema fundamental está en vencer el aislamiento impuesto por las distancias, en agrupar una población diseminada, en crear en el hombre de campo, individualista y solitario, el sentimiento de la solidaridad" (Castro, 1938: 6). La lectura de su texto permite afirmar que, para Castro, mirar a México era

Campesino". Conferencia dictada a las comunidades agrarias en San Luis de Potosí el 2 de noviembre de 1935; El sistema de las Escuelas Rurales en México". - Publicada por la -Secretaría de 1. Pública. (México 1927); Moisés Sáenz; "El Sistema de Escuelas Rurales en Méjico"; Censo de la Comisión Agraria Nacional; Bases dadas por la Secretaria de I. P. el 2 de febrero de 1927. (México); Lo que ha hecho el gobierno del Gral. Cárdenas en el ramo de I. P. (folleto gráfico. 1936); Las Misiones Culturales en 1927. (Memoria de la Secretaría do I. Pública); "Exposición Objetiva del Plan Sexenal" (D. A. P. P.) (Castro, 1938).

${ }^{8}$ En México "es muy pequeña la cantidad de trabajadores rurales que viven diseminados en el campo en oposición a lo que sucede en nuestro país. Tanto la hacienda como el ejido agrupan la población alrededor de un centro. Los trabajadores viven con sus familias en el poblado de la hacienda, a diferencia de los peones de nuestra campaña que viven lejos de los suyos, a veces a muchas leguas de distancia, o con ellos, en lugares apartados, difícilmente accesibles, los "puestos", dependencias de las grandes estancias" (Castro, 1938: 6). 
una forma de repensar su país y la educación en él. La descripción del "hombre de campo" como "individualista", "solitario" y no "solidario" es una caracterización que empezó a esbozar en esta Síntesis y que retomó en trabajos posteriores, principalmente en su libro La escuela rural en el Uruguay (1944), sirviéndole de sustento para pensar programas educativos para la gente del campo.

Según el autor, "el problema fundamental está en vencer el aislamiento impuesto por las distancias" y para enfrentarlo recomendó la lectura de un libro: "Orientado hacia la solución de este problema, ha escrito un maestro compatriota, Agustín Ferreiro, un libro que contiene valores excepcionales, titulado La enseñanza primaria en el medio rural (Castro, 1938: 6). Esta referencia al libro de Ferreiro, publicado un año antes de la escritura de esta Síntesis, da cuenta de un proceso colectivo de construcción pedagógica por parte de los maestros uruguayos; que un educador haya citado a otro indica una circulación de ideas entre pares sobre una preocupación común. Vale recordar que este proceso venía gestándose desde comienzos de la década de 1930; un evento importante fue el Congreso Nacional de Maestros, celebrado en 1933 en Montevideo, en el cual participó Ferreiro en su rol de inspector escolar. Ahí se reunió una comisión bajo la pregunta "¿Qué puede hacer la escuela para evitar la despoblación del campo?". Ferreiro y otras maestras y maestros expusieron argumentos a favor de la ruralización de la escuela y tres años después, para un Concurso de Pedagogía escribió el libro citado por Castro. En otro apartado de esta Síntesis, titulado "Hacia la ruralización", el autor señala que "México va así a la vanguardia de un movimiento que se inicia en estos momentos en materia de educación, orientado a reivindicar la especificidad, diríamos, de la escuela rural" (1938: 24). Ir a la vanguardia significaba que había otros países que iban detrás, que lo seguían. En este sentido, Castro explicó que el fenómeno de "creciente urbanismo y la inquietante despoblación de la campaña" era mundial y que además de las causas económicas que lo generó, la huida del campo era "consecuencia, en no pequeña escala, del régimen educacional que ha orientado la enseñanza pública en todo el mundo" (1938: 24), un régimen educativo no ruralista, pensado para los habitantes de las ciudades.

En esta defensa de la ruralización de la educación Castró apeló no sólo a México como "vanguardia" de un movimiento internacional, sino que también mencionó la 5ta Conferencia Internacional Bureau International d’Educatión, la cual estuvo dedicada a estudiar el fenómeno de despoblación de los campos y donde los 43 países participantes firmaron un documento en favor de ruralización de la educación como una posible solución a este problema. Y, por último, se refirió además a la existencia de Escuelas Normales Rurales en otros países. Estas tres menciones pueden interpretarse como una búsqueda de legitimación en el escenario internacional de su propia posición en favor de esta tendencia. A su vez, resulta necesario inscribir la defensa de esta posición en el contexto de producción de este documento; en Uruguay, en la década de 1930, comenzó la discusión sobre cómo debía ser la 
educación en el campo por parte maestros, inspectores y demás agentes gubernamentales, dividiéndose las posiciones entre quienes estaban a favor de que la escuela rural tuviera una orientación específica y quienes estaban en contra de esta diferenciación, siendo Castro y Ferreiro dos de los principales impulsores de esta reforma y la maestra Reina Reyes, quien en 1943 había ganado el primer premio en el Concurso de Pedagogía con su trabajo La escuela rural que el Uruguay necesita, una de las representantes de la oposición.

La polémica continuó hasta 1949, año en que los maestros rurales, con el aval del Gobierno Nacional de Luis Batlle Berres, crearon el Programa de Escuelas Rurales. La posición a favor de la pedagogía ruralista terminó imponiéndose en esta nueva legislación. Tras la aprobación de este programa, Julio Castro escribió para Semanario Marcha respecto de esta disputa, esgrimiendo que "Entre los maestros el asunto ha sido discutido por años: unos querían darle a la escuela rural características particulares en función de su ambiente de actividades. Si la escuela va a ser para el campo, lo lógico es que en el campo tome su configuración" (Castro, 2013: 124). Consideraba que, hasta entonces, la escuela urbana y la escuela rural habían sido "la misma cosa. La escuela rural no es más que un salón escolar cualquiera transportado al medio del campo. Vive allí y vegeta porque no tiene derivaciones hacia las actividades que a su alrededor se realizan" (Castro, 2013: 124). Según el maestro y periodista, los argumentos de quienes estaban a favor de esta reforma educativa sostenían que "La escuela rural debía ruralizarse, tomar contacto con el mundo de alrededor e influir, en la medida de lo posible, en el mejoramiento de la zona" (Castro, 2013: 124). Mientras que quienes se oponían, que por mucho tiempo representaron la opinión oficial del Consejo de Educación, consideraban que "No hay dos sociedades, ni dos culturas. Y el hombre es el mismo en el campo que en la ciudad. La educación debe atender al hombre y ese hombre debe ser el mismo en todas partes. Una escuela rural distinta de una escuela urbana, tiende a dividir la sociedad en dos sectores: uno campesino y otro ciudadano. Tiende, además, una escuela rural orientada hacia la ruralización, a atar el destino del hombre, a fijarlo en un punto" (Castro, 2013: 124). Castro, en las antípodas de esta posición, pretendía que hubiera políticas educativas diferenciales para las escuelas del campo, no una adaptación en la oferta curricular de la ciudad para el área rural, sino cambios generales en las finalidades, metodologías, contenidos, así como también en la concepción misma de escuela. ${ }^{9}$ Estas citas dan cuenta de un largo proceso de discusión en el ámbito de la educación pública en Uruguay

\footnotetext{
${ }^{9}$ Previo al Programa de Escuelas Rurales de 1949, las escuelas primarias de los campos estaban regidas por un Programa sancionado en 1917. Según Castro, las diferencias entre el programa rural de 1917 y el asignado a las escuelas urbanas son "diferencias de "dosis", de exigencias, de jerarquización de asignaturas. [...] Ello se debe a que hasta ahora no se le ha asignado a la escuela rural una función específicamente propia, y la concepción diferencial sólo se reduce a un problema de adaptación de un contenido común" (Castro, 1944: 97).
} 
y revelan un problema pedagógico que se generó a nivel mundial con la implementación y expansión de los sistemas educativos nacionales: el intento de homogeneizar a una población diversa mediante la implementación de un programa o curriculum único. Esta imposición generalmente fue defendida con el argumento de la igualdad en la oferta educativa para todos los ciudadanos, entendida como garantía de justicia pedagógica, en oposición a un programa educativo diferenciado según los contextos socioeconómicos y geográficos. Hacer programas educativos diferenciados según los contextos implicaría admitir la diversidad existente dentro de un territorio nacional; que haya diferentes ofertas educativas en función del contexto es, muchas veces, interpretado como una desigualdad en las oportunidades educativas.

En las décadas de 1930 y 1940 el problema no era sólo curriculum único o diferenciado, sino también la centralización/descentralización de la educación y la participación del "vecindario" y demás actores locales en la administración de los locales escolares. Al respecto, el mismo Julio Castro, haciendo una historización de la "Escuela Rural en Uruguay", explica que, el ideólogo del sistema educativo de su país, José Pedro Varela, le daba mucho valor a la educación en el campo e "intento hacer de la escuela rural un centro de cultura popular" (Castro, 1944: 53). Varela pretendía que las escuelas tuvieran "Comisiones de distrito" que las administre y designe al personal docente. Sin embargo, la legislación que finalmente se implementó, no tuvo en cuenta este aspecto del proyecto valeriano y tendió hacia la centralización administrativa y pedagógica de las escuelas, quedando "la misión del vecindario" limitada "a la intervención, por cierto, muy retaceada, de las Comisiones Pro Fomento y nada más. Con esta centralización sistemática, la escuela fue tomando las características de un instituto de desanalfabetización" (Castro, 1944: 54).

Esta reducción de la función de la escuela rural a una unidad "desanalfabetizadora" da cuenta de que, más allá de las pretensiones formales de brindar una educación común en el campo y en la ciudad, en la vida escolar cotidiana, las prácticas de enseñanza y de aprendizaje eran y son distintas, porque las condiciones para las mismas lo son también. No es igual enseñar en una escuela de personal único, a niños y niñas de distintas edades todos juntos en el mismo salón, que enseñar en una escuela urbana, en la cual cada maestro tiene a cargo un grado; como tampoco, son las mismas las expectativas, intereses, saberes y posibilidades en relación a la educación escolar que tienen los niños, niñas y sus familias, en uno y en otro contexto.

Con este análisis no se está justificando ni argumentando a favor de la reducción de las escuelas de los campos a la enseñanza únicamente de la lectoescritura, ya que justamente esto es lo que los maestros rurales uruguayos denunciaban y querían modificar con sus 
proyectos ruralistas, sino que simplemente se demuestra que la existencia de un plan de estudios común o similar no es garantía de una enseñanza igualitaria en el campo y la ciudad.

\section{La educación: entre el control estatal y la participación popular en el financiamiento de las escuelas y demás proyectos educativos}

Una de las comparaciones que realizó Castro en Síntesis tiene que ver con la participación popular en el financiamiento de las escuelas y demás proyectos educativos. El autor señaló que en México, debido a la densidad de población, en muchos lugares, a pesar de que la inversión pública en educación desde 1920 aumentó año tras año, el déficit en cuanto a cobertura no se alcanzó a cubrir y que, en muchos casos, el sostenimiento económico de las escuelas, su construcción, incluso la alimentación del maestro, fueron solventadas por las comunidades. ${ }^{10}$ Describió la educación rural mexicana y la analizó políticamente advirtiendo los riesgos de "burocratización" que implicaba todo proceso de concentración poder político. A su vez, comparó el desarrollo del sistema educativo uruguayo con el mexicano, dando cuenta de cómo el primero logró a fines de siglo XIX crear una red de escuelas primarias suficientes para toda la población, población considerablemente menor que la mexicana, siendo la educación gratuita para los ciudadanos y financiada por el Estado, a diferencia del proceso histórico mexicano, país en el cual recién después de 1920 comenzó a masificarse la enseñanza primaria con la creación de las escuelas federales. Sin embargo, este desarrollo de la educación básica posterior a la Revolución mexicana se realizó mediante un amplio proceso de participación popular (misiones culturales, campañas "desanalfabetización", distribución gratuita de libros, etc.), que pretendió hacer que las escuelas no fueran solamen-

10 "Se necesitarán por lo menos 30.000 escuelas más para llenar las necesidades de la población rural [...] Las posibilidades económicas, no le permiten aún al país este gigantesco esfuerzo. Tal vez la solución esté en la frase de Frank Tannenbaum, que transcribimos: 'La comunidad rural debe sostener a la escuela en el futuro en la misma forma que sostuvo a la Iglesia en el pasado'. Y agregamos nosotros, tal vez sea esa la única forma de que la escuela no pierda su carácter revolucionario, no se haga indiferente al movimiento, no se burocratice, como sucede con las actividades que sostiene el Estado [...] La escuela rural mexicana está sostenida por una mística de redención; si perdiera un día esa fuerza que le dio origen y fisonomía perdería sus más valiosas posibilidades de futuro [...]. En nuestro país la gratuidad de la enseñanza es cosa resuelta, sobre la cual no hay discusión. Sin embargo, hay que reconocer que el movimiento de las Universidades Populares que se caracteriza por ser [...] un movimiento netamente popular, con cierta búsqueda de redención por la cultura, está totalmente financiado por el pueblo, sin subvenciones oficiales, ni de partidos, ni de gobiernos extranjeros, como estúpidamente creen algunos. Además, bueno es recordarlo, idéntico espíritu fue el que presidió la obra de la Sociedad de Amigos de la Educación Popular en la Reforma de José Pedro Varela." (Castro, 1938: 20). 
te una agencia del Estado sino, también, "Casas del Pueblo", es decir, centros de participación y organización comunal.

Castro comparó la participación popular en el sostenimiento de las propuestas educativas mexicanas con el Movimiento de Universidades Populares uruguayo (1931-1942), movimiento que se generó en un contexto político conservador, desarrollado inicialmente sin el apoyo del Estado, pero como una estrategia para ampliar y democratizar la educación superior siguiendo el ideario de educación popular de José Pedro Varela. ${ }^{11}$ Las universidades populares uruguayas formaron parte del proceso latinoamericano de organización del movimiento estudiantil de principios del siglo Xx con las reformas universitarias y los primeros congresos latinoamericanos de estudiantes (Moraga, 2014).

Este movimiento uruguayo constituyó un antecedente de otros proyectos educativos que implicaron la organización autónoma de estudiantes y docentes como lo fueron las misiones sociopedagógicas en la década de 1940 y el ICER (Instituto Cooperativo de Educación Rural) ¿a partir de 1961 (Scagliola, 2016 y Bordoli, 2009). En este sentido se puede afirmar que entre 1920 y 1960 se conformó un cuerpo social heterogéneo, compuesto por maestros, intelectuales, profesores universitarios y estudiantes que asumió un rol activo en la construcción de proyectos educativos para los sectores populares en el campo y en la ciudad, muchos de los cuales terminaron oficializándose, reconfigurándose y formando parte de la educación pública.

\section{Las misiones sociopedagógicas y el Programa de Escuelas Rurales en Uruguay}

Este artículo se inicia con el análisis el documento Síntesis del proceso educacional mexicano, escrito por el uruguayo Julio Castro en 1938, en el cual se manifiesta que México constituyó la vanguardia de un movimiento mundial que sostenía la necesidad de ruralizar la educación en los campos. En la reconstrucción de la trayectoria del autor y de sus publicaciones se puede identificar cómo su vínculo con México fue creciendo llegando incluso a vivir y trabajar allí entre 1952 y 1954.

Luego de la Sintesis el siguiente texto publicado en el cual el maestro hizo referencia a México es en la nota periodística "Lo que el alegre fin de año oculta" (1942), en el cual menciona los casos mexicano, chileno y el de la Republica Española como ejemplos de mo-

\footnotetext{
11 "Éstas comenzaron como una creación del Centro de Estudiantes ARIEL fundado en 1917, que publicó una revista con su nombre bajo la dirección de Carlos Quijano, su presidente hasta 1924 (Equipo de Investigación de Historias Universitarias, 2018).
} 
vimientos educacionales y exhorta a los uruguayos a "reaccionar contra el abandono escolar" (Castro, 1942: 16). Dos años más tarde, en el libro La escuela rural en el Uruguay (1944), siguió comparando, sin pecar de ingenuo o idealista, la educación de México con Uruguay con la intención de comprender y aprender, más que de imitar.

En 1945 se realizó en Uruguay por primera vez una misión sociopedagógica la cual se realizó en el poblado de Caraguatá ubicado en el departamento de Tacuarembó. Fue organizada por estudiantes del magisterio de Montevideo y Julio Castro participó como profesor acompañante. Luego de esta primera misión, Castro publicó en la contraportada de Marcha una nota sobre la misma, en la cual imbricó una vez más su rol de periodista con el de educador con el objetivo de ejercer presión política para generar cambios en la sociedad y en el gobierno en favor de los habitantes del campo. Allí denunció las malas condiciones de vida de los habitantes de Caraguata y manifestó: "lo visto allá debe saberlo todo el país [...] no hay derecho a seguir ignorando que sucede entre nuestra gente, sin que, en buena parte, nos convirtamos en culpables de un estado de cosas por la tozudez egoísta de seguirlo ignorando" (Castro, 1945).

En la nota explica además que las misiones no fueron una invención uruguaya y cita el caso de México, España y Venezuela; consideraba que esta primera experiencia en su país había sido "demasiado cultural" y que "con cultura, solo en campaña, no hacemos nada" (Castro, 1945). Este señalamiento estaba seguramente sostenido en el conocimiento de que en las misiones culturales mexicanas participaron profesionales y estudiantes de distintas áreas (salud, agronomía, educación, etc.) y que su función excedía la difusión cultural.

En 1947 volvió a acompañar a los estudiantes en otra misión, y en 1948 viajó por primera vez a México tras ser invitado a participar del Congreso Nacional de Escuela Rural Mexicana, donde integró la comisión redactora del informe final. Aprovechó su viaje para adelantarse en las comunidades campesinas y conocer en persona aquello sobre lo cual venia leyendo hacía años. Luego de regresar a su país, visitó distintos países latinoamericanos, y tras su retorno a Montevideo, los días 20 y 27 de octubre dictó una serie de conferencias organizada por la Comisión de Cultura de la Asociación de Bancarios del Uruguay denominadas "Cómo viven 'los de abajo' en los países de América Latina" (1948), en las cuales dedicó una gran parte del tiempo a describir su experiencia en México, el registro de su recorrido por distintas comunidades indígenas y su visión sobre la relación entre los pobladores, las escuelas y el trabajo de los maestros rurales.

Dos meses después de haber brindado esas conferencias, entre 2 al 6 de enero de 1949, se realizó en Piriápolis un Congreso de Maestros Rurales convocado por el Consejo Nacional de Enseñanza Primaria y Normal para la construcción conjunta de un Programa de Escuelas Rurales. Castro fue uno de los organizadores del congreso y trabajó en la comisión redacto- 
ra del programa. En el texto del programa se pueden identificar distintas ideas y conceptos basados en experiencias educativas mexicanas, siendo el concepto de escuela como "Casas del Pueblo" uno de los centrales. ${ }^{12}$

Los vínculos del maestro con México se fortalecieron después de su primer viaje en 1948. Entre 1952 y 1954 vivió en dicho país para ocupar el cargo que la UNESCO y el gobierno mexicano le confiaron como Subdirector del Centro Regional de Educación Fundamental para la América Latina (CREFAL). En 1954 volvió a Uruguay, se jubiló de sus cargos docentes y continuó realizando diversos informes sobre educación en distintos países de la región. En 1964 viajó a México nuevamente con una delegación de 25 educadores uruguayos para participar en la Asamblea Mundial de Educación, organizada por la Academia Mexicana de la Educación y la Liga Internacional de la Enseñanza, la Educación y la Cultura Popular. Presidió la Segunda Comisión de "Problemas de la Educación en América Latina" e integró la comisión redactora de la memoria final. Ese mismo año dictó con el maestro y escritor uruguayo Jesualdo Sosa un ciclo de conferencias en distintas ciudades mexicanas y en 1965 colaboró en un curso sobre planeación educativa y brindó conferencias sobre ese mismo tema (Soler, 2007), recorrido que da cuenta del reconocimiento que tuvieron estos maestros en México también.

Estas interconexiones revelan cómo las historias se cruzan y entretejen por lo bajo, nutriendo nuevas formaciones, producciones, configuraciones, prácticas y sentidos. La Síntesis del proceso educacional mexicano puede entenderse como el inicio de una inquietud que lleva a Castro a conocer más sobre la educación y la vida en el México posrevolucionario. Ubicarlo como un documento más en el análisis de su trayectoria da cuenta de un conjunto de motivaciones y preocupaciones que con los años Julio Castro pudo traducir en acción y producción.

\section{Conclusión}

En cuanto a la metodología de investigación, el análisis de las trayectorias de actores claves en procesos sociales y políticos posibilita identificar intercambios que en estudios de rela-

\footnotetext{
12 Esta similitud de ideas y terminologías fue advertida por militares uruguayos; en 1978, un año después de que el ejército uruguayo secuestró y asesinó a Julio Castro, el Comando General del Ejército Uruguayo publicó el libro Testimonio de una nación agredida, que incluía un informe sobre la "infiltración en la enseñanza", en el que se detalla que "Hacia 1949, una nueva circunstancia termina impulsando esta ofensiva de penetración, que por entonces aparecía inadvertida para la inmensa mayoría de los uruguayos. Son aprobados los programas de las Escuelas Rurales, realizados por Julio Castro que copió capítulos enteros de los programas mexicanos". Comando General del Ejército, 1978, p. 218.
} 
ciones entre países parecen invisibles ya que no se dan por la vía oficial. En este caso, la reconstrucción de la vida de maestros e intelectuales como Julio Castro permite visibilizar una red de interconexiones entre diversos actores, instituciones, ideas y experiencias pedagógicas en la región que configuraron, entre 1930 y 1960, una nueva pedagogía en Uruguay.

Como conclusión planteamos la potencialidad de producir investigaciones en historia de la educación que trasciendan las fronteras nacionales y que den cuenta de las conexiones en donde se configuran saberes y proyectos pedagógicos. Queda como tarea pendiente continuar profundizando en el estudio de las pedagogías ruralistas latinoamericanas, sus invenciones, experiencias, contradicciones y conflictos. En el caso de Uruguay es para desatacar el protagonismo de los maestros en el desarrollo de un movimiento pedagógico (1930-1960), que dentro del ámbito de la educación pública, replantearon las relaciones entre la escuela y el medio en que se inserta, el sentido y el alcance de las prácticas educativas allí situadas, así como también reconstruyeron el trabajo docente, trascendiendo la tarea "desanalfabetizadora", colocándose como sujetos promotores del desarrollo local desde una perspectiva integral, para la cual se formaron específicamente, en vinculación constante con otras experiencias de educación latinoamericanas, particularmente, la mexicana.

Una de las tensiones principales que plantearon las pedagogías ruralistas durante la primera mitad del siglo Xx, como discusión pedagógica y de política educativa, tiene que ver con el desarrollo de proyectos educativos distintos para los habitantes del campo y de la ciudad. En Uruguay la educación pensada desde y para el contexto urbano era vista como una imposición estéril en el campo, pero, a su vez, las propuestas de educativas para el entorno rural eran consideradas como algo que podría estigmatizar y "atar" al "campesino" a su entorno. Este debate adquiere otro tamiz en sociedades como la mexicana donde quienes vivían en el campo eran predominantemente indígenas y mestizos, lo cual introduce otros elementos a considerar como la diversidad cultural y lingüística. Estas tensiones provocan preguntas tan básicas como: ¿La enseñanza debe ser la misma en todas las escuelas del país?, ¿es posible esto?, ¿por qué?, ¿para qué?, ¿a quiénes beneficia?

La relación entre escuela y "medio" y "contexto" ha sido objeto de debate pedagógico permanente en Uruguay, pasando actualmente la atención en la pobreza urbana (Martinis y Redondo, 2006, Romano, 2006, Bordoli, 2009). Así como en 1933 se preguntaban en un Congreso Nacional de Maestros, qué puede hacer la escuela para evitar la despoblación del campo, cuál son sus alcances y sus límites, ahora se preguntan qué puede hacer la escuela en contextos de pobreza urbana, cuál es su función y sentido en esos contextos, cómo debe ser el trabajo docente y la relación con los estudiantes y sus familias (Reisin, 2017). 


\section{Fuentes}

Bibliografía

Alonso, María y Gabriel Scagliola (coords.) (2012), Misiones sociopedagógicas del Uruguay (1945-1971), Documentos para la memoria, Consejo de Formación en Educación (ANEP), Montevideo.

Ansaldi, Waldo y Verónica Giordano (2012), América Latina. La construcción del orden, t. I y II, Ariel, Buenos Aires.

Bordoli, Eloisa (2009), "La extensión universitaria como una experiencia de 'comunidad' educativa", en Antonio Romano y Eloisa Bordoli (comps.), Pensar la escuela como proyecto (político) pedagógico, Psicolibros Waslala, Montevideo, pp. 25-40.

Calderón Mólgora, Marco Antonio (2018), "México: de la educación indígena a la educación rural", Revista Historia y Memoria de la Educación, núm. 7, pp. 153-190.

Castro, Julio (1942) "Lo que el alegre fin de año oculta", Marcha, núm. 167, Montevideo, diciembre, p. 16.

Civera Alicia, Juan Alfonseca y Carlos Escalante (coords.) (2011), Campesinos y escolares: la construcción de la escuela en el campo latinoamericano (siglos xix yxx), Miguel Ángel Porrúa, El Colegio Mexiquense, México, pp. 221-262.

Civera, Alicia (2008), La escuela como opción de vida: la formación de maestros normalistas rurales en México 1921-1945, El Colegio Mexiquense, México.

Civera, Alicia (2011), "Alcances y retos de la historiografía sobre la escuela de los campos en América Latina (siglos XIX y XX)", Cuadernos de historia, núm. 34, Santiago de Chile, Universidad de Chile, junio, pp. 7-30.

Civera, Alicia y Antón Costa Rico (2018), "Desde la historia de la educación: educación y mundo", Revista Historia y Memoria de la Educación, núm. 7, Facultad de Educación, Islas Canarias, pp. 9-45.

Civera, Alicia y Lucia Lionetti (2010), "La educación rural en América Latina siglos XIX-XX", Naveg@ mérica. Revista electrónica editada por la Asociación Española de Americanistas, núm 4 (dossier).

Consejo Nacional de Enseñanza Primaria y Normal. República Oriental del Uruguay (1950) "Programa para Escuelas Rurales", Imprenta Nacional, Montevideo.

Demarchi, Marta y Marcia Rivera (1999), La educación rural en el Uruguay: construcción y vigencia de una doctrina, Departamento de Publicaciones FHCE, Montevideo.

Demarchi, Marta y Marcia Rivera (2014), Miguel Soler Roca. Educación, resistencia y esperanza, CLACSO, Buenos Aires.

Díaz Estévez, Pablo y Víctor Díaz Estévez (2019), "El 'campo' como principio educativo. Aportes desde una perspectiva intercultural crítica en Uruguay", en Daniel San Martín Cantero (coord.), Experiencias latinoamericanas para re-pensar la educación 'rural', CIED Facultad de Educación, Universidad Católica de Temuco, Chile, pp. 125 -139.

Espeche, Ximena (2016), La paradoja uruguaya. Intelectuales, latinoamericanismo y nación a mediados del siglo XX. Ediciones Universidad Nacional de Quilmes, Buenos Aires.

Loyo, Engracia (1999) Gobiernos revolucionarios y educación popular en México, 1911-1928, El Colegio de México, México.

Martinis, Pablo y Patricia Redondo (comps.) (2006), Igualdad y educación. Escrituras (entre) dos orillas, Del Estante Editorial, Buenos Aires.

Moraga Valle, Fabio (2014), "Reforma desde el sur, revolución desde el norte: El Primer Congreso Internacional de Estudiantes de 1921", Estudios de Historia Moderna Y Contemporánea de México, núm. 47, México, junio, pp. 155-195. 
Moraga Valle, Fabio (2016), "Las ideas pedagógicas de Tolstoi y Tagore en el proyecto vasconcelista de educación, 1921-1964", Historia Mexicana, vol. 65, núm. 3, El Colegio de México, México, enero, pp. 1341-1404.

Moraga Valle, Fabio (2018), "Educación, exilio y diplomacia: Vasconcelos, Mistral, Torres Bodet y la proyección internacional de sus ideas educativas, 1921-1964", Revista de Historia de América, pp. 61-94.

Palacios, Guillermo (1999), La pluma y el arado. Los intelectuales pedagogos y la construcción sociocultural del "problema campesino" en México, 1932-1934, El Colegio de México, México.

Pita González, Alejandra (2014) Educar para la paz: México y la cooperación intelectual internacional, 1922 1948, Secretaría de Relaciones Exteriores, Universidad de Colima, México.

Reisin, Pamela (2017) “¿Cómo acompañar las trayectorias de niños y niñas en las escuelas públicas? La experiencia del Programa de Maestros Comunitarios en Uruguay", Revista Síntesis, núm. 7/2016, Editorial Facultad de Filosofía y Humanidades UNC, pp. 76-86.

Reyes, Reina (1943), "La escuela rural que el Uruguay necesita", Anales de Instrucción Primaria, Montevideo.

Rockwell, Elsie (2018)[2005] "Bardas, cercos y llaves: El encierro de escuelas indígenas rurales", en Nicolás Arata, Carlos Escalante y Ana Padawer, Elsie Rockwell: Vivir entre escuelas: Relatos y presencias, CLACSO, Argentina: pp. 355-390.

Rockwell, Elsie (2007), Hacer escuela, hacer Estado: la educación posrevolucionaria vista desde Tlaxcala, El Colegio de Michoacán-CIESAS, México.

Romano, Antonio (2006) "Lo que no tiene nombre, la pobreza en educación" en Martinis Pablo y Redondo Patricia (comps.), Igualdad y educación. Escrituras (entre) dos orillas, Del Estante Editorial, Buenos Aires, pp. 141-162.

Santos, Limber (2012), "La pedagogía rural hoy: una actualización necesaria", Revista Quehacer educativo, núm. 116, FUM-TEP, Montevideo, diciembre, pp. 9-15.

Soler Roca, Miguel (1987), "El movimiento a favor de una nueva escuela rural", en Ana María Angione et al., Dos décadas en la historia de la escuela uruguaya. El testimonio de los protagonistas, Edición de la Revista de la Educación del Pueblo, Montevideo.

Soler Roca, Miguel (1996), Educación y vida rural en América Latina, Federación Uruguaya del Magisterio, Instituto del Terer Mundo, Montevideo.

Vaughan, Mary Kay (2000), Política cultural en la Revolución mexicana: maestros, campesinos y escuelas en México, 1930-1940, Fondo de Cultura Económica, SEP, México.

Werle, Flávia y Ana Maria Carvalho (2009) "A educação para a zona rural no sul do Brasil: sentidos e perspectivas a partir das conferências brasileiras de educação", en Teresa González y Oresta López (coords.), Educación rural en Iberoamérica. Experiencia histórica y construcción de sentido, Anroat, España, pp. 79-108.

Werle, Flávia (comp.) (2007), Educacao rural em perspectiva internacional. Intituicoes, practicas e formazao do prodessor, Editora Uniji, Brasil.

\section{Recursos electrónicos}

Betemps Bozzano, Caroline (2003), "Julio Castro: periodismo y acción transformadores", Seminario Taller de Análisis de la Comunicación, Licenciatura en Ciencias de la Comunicación. Universidad de la República, documento (html o pdf) disponible en: <https://www.academia.edu/8239370/Julio_Castro._Periodismo_y_acci\%C3\%B3n_transfrmadores (fecha de consulta: 17/04/2019). 
Castro, Julio (1938) Síntesis del Proceso Educacional Mexicano, Oficina de Prensa de Periodistas Libres, documento (pdf) disponible en: <http://www.autoresdeluruguay.uy/biblioteca/julio_castro/lib/exe/ fetch.php?media=sintesis_del_proceso_educacional_mexicano.pdf(fecha de consulta: 17/04/2019).

Castro, Julio (1944) La escuela rural en el Uruguay, Talleres Gráficos "33", Montevideo, documento (htm o pdf), disponible en: <http://www.autoresdeluruguay.uy/biblioteca/julio_castro/lib/exe/fetch. php?media=la_escuela_rural.pdf(fecha de consulta: 17/04/2019).

Castro, Julio (1945), "La misión pedagógica de los alumnos normalistas", Marcha, núm. 289, Montevideo, julio, documento (pdf), disponible en: <http://www.autoresdeluruguay.uy/biblioteca/julio_castro/ lib/exe/fetch.php?media=la_mision_marcha_n_289_6_07_1945.pdf (fecha de consulta: 17/04/2019).

Castro, Julio (2008), "Cómo viven 'los de abajo' en los países de América Latina", Ministerio de Relaciones Exteriores, Consejo de Educación Técnico Profesional, Universidad del Trabajo del Uruguay, Serie Edición Homenaje, vol. 17, documento (pdf) disponible en: <http://www.autoresdeluruguay. uy/biblioteca/julio_castro/lib/exe/fetch.php?media=como_viven_los_de_abajo_en_los_paises_ de_america_latina_2008.pdf (fecha de consulta: 9/03/2017).

Castro, Julio (2013), Palabras de Julio. Selección de textos, Ministerio de Educación y Cultura, Montevideo, documento (pdf) disponible en: <http://www.autoresdeluruguay.uy/biblioteca/julio_castro/lib/exe/ fetch.php?media=palabras_de_julio_seleccion_de_textos.pdf (fecha de consulta: 17/04/2019).

Comando General del Ejército (1978), "Testimonio de una Nación agredida", Montevideo, documento (pdf) disponible en: http://radiovirtualga.org.uy/Libros/Nueva\%20carpeta/Testimonio\%20de\%20 una\%20nacion\%20agredida.pdf (fecha de consulta: 20/04/2019).

Santos, Limber (2018), "El pensamiento pedagógico nacional a través del Movimiento de Educación Rural", Revista Quehacer Educativo, Montevideo, documento (audiovisual) disponible en: https:// www.youtube.com/watch?v=MV4UV0xCIfA\&feature=youtu.be (fecha de consulta: 16/05/2019).

Scagliola, Gabriel (2016), "Dos iniciativas estudiantiles: misiones sociopedagógicas y universidades populares en Uruguay (1931 - 1956)", documento (html) disponible en: <https://docplayer. es/35032491-Dos-iniciativas-estudiantiles-misiones-socio-pedagogicas-y-universidades-populares-en-uruguay.html (fecha de consulta: 10/12/2018).

Soler Roca, Miguel (2007), "Cronología- extraído del homenaje a Julio Castro realizado por el MEC", Barcelona, documento (pdf) disponible en: http://www.autoresdeluruguay.uy/biblioteca/julio_castro/lib/exe/fetch.php?media=cronologia_juliocastro.pdf (fecha de consulta: 10/12/2018).

Taracena Arriola, Arturo (2006), "Descubrir América en Europa: la asociación general de estudiantes latinoamericanos de París (1925-1933)", en Calvo, T., A. Musset (eds.), Des Indes occidentales à l'Amérique Latine, vol. 2, Centro de Estudios Mexicanos y Centroamericanos, documento (html o pdf) disponible en <:https://books.openedition.org/cemca/2126?lang=en\#authors (fecha de consulta: 17/04/2019).

Pamela Ruth Reisin es becaria doctoral de conicet en el Centro de Estudios Avanzados, Facultad de Ciencias Sociales, Universidad Nacional de Córdoba, Argentina. Licenciada y profesora en Ciencias de la Educación por la Universidad Nacional de Córdoba. Sus líneas de investigación son la historia de la educación en Latinoamérica, educación rural y pedagogías para la inclusión educativa. Entre sus publicaciones recientes se cuentan: "La metodología de 'historias conectadas' para el estudio de la configuración de movimientos pedagógicos. El caso de la pedagogía ruralista y sus conexiones con la educación rural mexicana ente 1930-1960", ponencia presentada en el II Workshop de Historia(s) de la 
educación. Itinerarios y debates hacia una perspectiva regional del campo, ICSOH CONICET, Universidad Nacional de Salta, 26 de abril de 2019; "El trabajo de los maestros comunitarios de Uruguay y sus conexiones con las pedagogías ruralistas de América Latina entre 19201940, 2017; Educación y equidad en contextos rurales: debates y propuestas, Red Temática de Investigación en Educación Rural (RIER-Conacyt) Facultad de Filosofía. Universidad Autónoma de Querétaro, México (en prensa).

Recibido: 5 de septiembre de 2019

Aceptado: 28 de noviembre de 2019 